\title{
Palliative treatment for breast cancer with cutaneous metastases - a case report
}

\author{
Nicolae Bacalbaşa ${ }^{1}$, Irina Bălescu², Vladislav Braşoveanu ${ }^{3}$, Dana Tomescu ${ }^{4}$ \\ Corresponding author: \\ Nicolae Bacalbaşa, MD \\ No. 2, Dimitrie Racoviță Street, \\ Bucharest, Romania \\ E-mail: nicolae_bacalbasa@yahoo.ro \\ 1"Carol Davila" University of Medicine and Pharmacy, Bucharest, Romania \\ 2"Ponderas" Hospital, Bucharest, Romania \\ 3"Dan Setlacec" Center of Gastrointestinal Disease and Liver Transplantation, \\ "Fundeni" Clinical Institute, Bucharest, Romania \\ ${ }^{4}$ Department of Anaethesia and Critical Care III, Fundeni Clinical Institute, Bucharest, Romania
}

\begin{abstract}
Skin metastases from breast cancer account for up to $20 \%$ of all cutaneous malignancies and are usually a sign of poor prognosis due to the fact that their presence is the sign that the underlying tumor has already infiltrated the lymphatics or the adjacent blood vessels. However, in all these cases the most efficient therapeutic approach remains neo-adjuvant chemotherapy followed by radical surgery. We present the case of a 43 year old patient diagnosed with ductal invasive breast cancer with cutaneous metastases in which this therapeutic protocol was successfully applied.
\end{abstract}

Key words: skin metastases, breast cancer, radical mastectomy

\section{INTRODUCTION}

Cutaneous metastases account for up to $9 \%$ of all distant metastases from various malignancies; although malignant melanoma is the most frequent carcinoma which metastasizes to the skin, in clinical practice the highest proportion of skin metastases originates from breast cancer (1-4). It has been demonstrated that skin metastases from breast cancer accounts for up to $20 \%$ of all cutaneous malignancies $(2,3,5-7)$. The presence of cutaneous metastases usually indicates that the underlying tumor has already infiltrated the blood vessels or the lymphatics and is considered as a poor prognosis sign (8).

\section{CASE REPORT}

We present the case of a 43-year-old patient who presented for the apparition of a cutaneous reddish, hemorrhagic lesion of almost $4 \mathrm{~cm}$ on the leftarm associated with a large tumoral mass at the level of the left breast which was observed by the patient almost one year before but it was neglected. The 
clinical examination revealed the presence of an indurated hemorrhagic and ulcerated area associated with the presence of a tumoral lesion with at the ipsilateral breast measuring $6 / 7 / 5 \mathrm{~cm}$ (figure 1). The lesion was painful and entirely modified the shape of the breast. The contralateral breast was normal while in the axillary area no palpable adenopathy was found. The imagistic studies confirmed the presence of a mammary tumor measuring $5 / 6 / 5 \mathrm{~cm}$. The lesion was biopsied and the histopathological study revealed the presence of a poorly differentiated ductal invasive carcinoma so the patient was referred to the oncology clinic where she was submitted to neo-adjuvant radiochemotherapy. After ending six cycles of Doxorubicin and cyclophosphamide she was submitted to Madden radical mastectomy; the cutaneous lesion was also excised (figure 2-4). The histopathological findings confirmed the result of the biopsy; ten of the 14 excised axillary lymph nodes were positive and five of them presented a ruptured capsula; the immunohistochemical studies showed the presence of estrogen receptors in up to $60 \%$ of tumor cells, progesterone receptors in $75 \%$ of tumor cells and the absence of HER2 neu; Ki 67 was present in up to $75 \%$ of tumor cells. The histopathological study of the skin lesion revealed the presence of anaplastic cells in the dermal structure with lymphatic emboli of large malignant cells. Postoperatively the patient was readdressed to the oncology clinic where she underwent adjuvant radiation therapy followed by hormone therapy with Tamoxifen.

\section{DISCUSSIONS}

Breast cancer continues to be a significant health problem worldwide, being the second most common malignancy in women (8). The presence of cutaneous metastases is more frequently seen in patients with breast cancer than in any other malignancy and is usually associated with poor prognosis especially due to the fact that their presence is in fact the sign that the malignant cells have already reached the systemic blood or lymphatic flow (9). While most skin metastases are thought to have hematogenous origin, cutaneous metastases from breast cancer seem to be more related to the lymphatic pattern of spread (10).

Most commonly cutaneous metastases from breast cancer are located on the chest skin although other locations such as abdomen, neck, upper limb and even scalp were reported $(11,12)$. In all these cases establishing the right diagnosis might be a real challenge due to the fact that breast cancer cutaneous

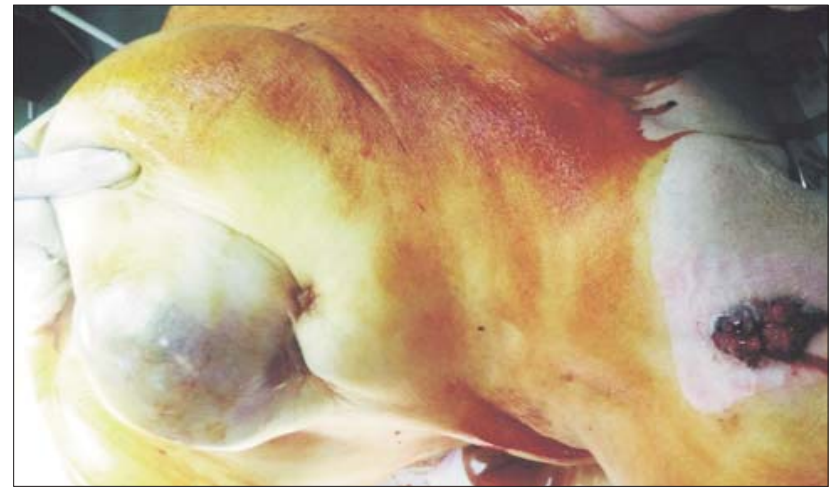

Figure 1 - Initial aspect: large tumor deforming the left breast associated with a hemorrhagic lesion on the left shoulder

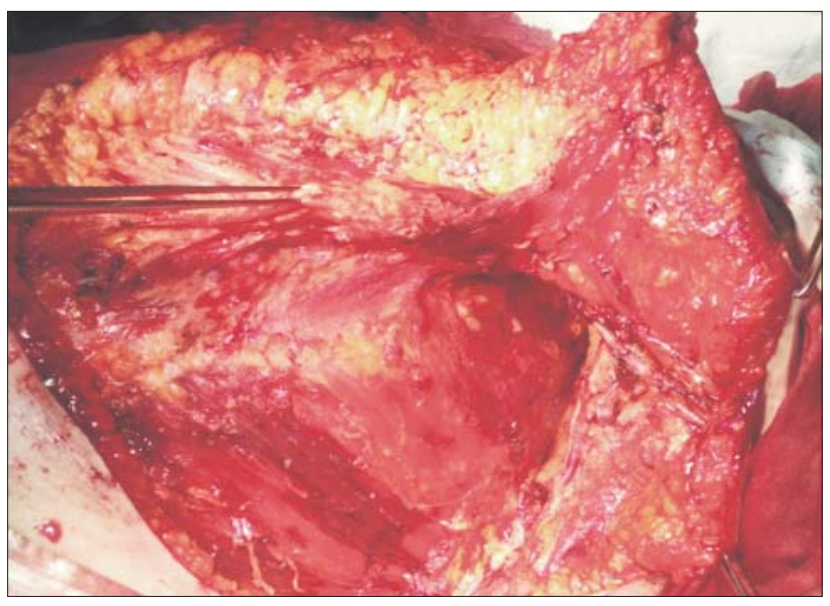

Figure 2 - The aspect after total radical mastectomy

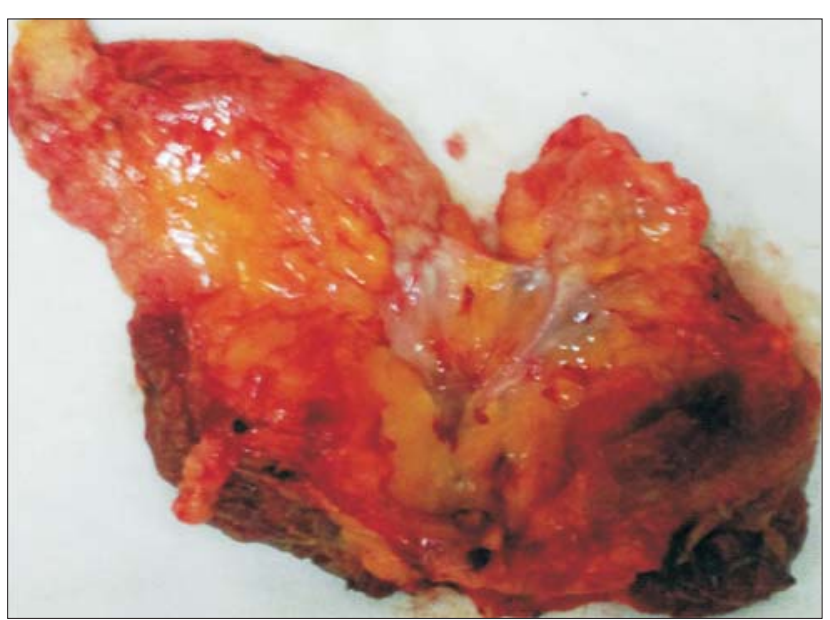

Figure 3 - The specimen of total radical mastectomy

metastases have a similar clinical aspect to other diseases such as lymphedema, cellulitis, targetoid lesions, erysipel or even radiation dermatitis (13-16). Macroscopic aspect can widely vary between indurated cutaneous lesions, erythematous solid papules or tiny ulcerated skin (11). Other local modifications might 


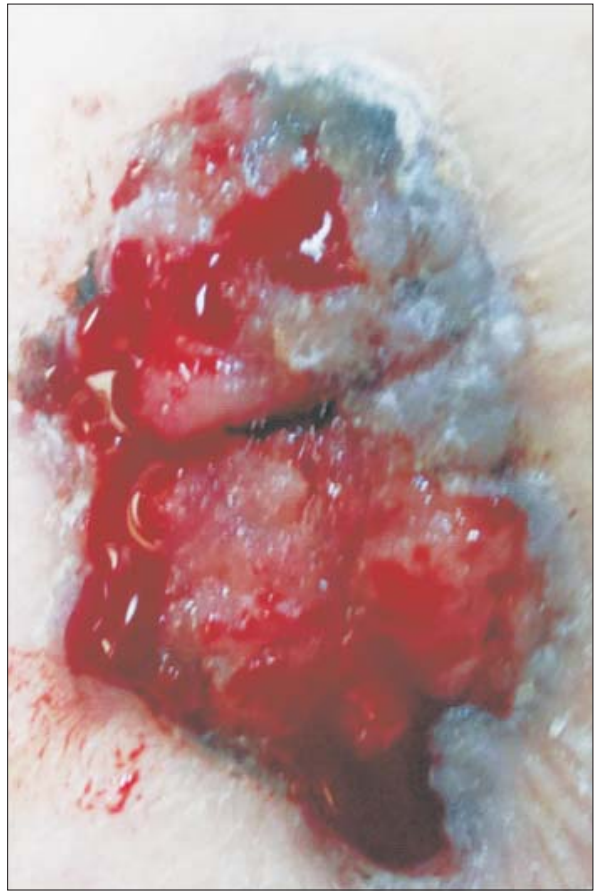

Figure 4 - Resection of the cutaneous lesion

suggest the presence of a carcinoma erysipeloides, carcinoma telangiectatum or carcinoma en cuirasse (17). Carcinoma erysipeloides is an inflammatory skin metastases accounting for less than $1 \%$ of the total metastases and is usually associated with the intraductal histopathological subtype (18). It presents sharply demarcated erythematous plaques and patches; the microscopical studies reveal the presence of malignant cells predominantly in the dermal vessels. The mechanism of formation of these lesions consists of the apparition of tumor emboli which obstruct the lymphatic vessels and produce a lesion with erysipeloid aspect (10). Another aspect of the cutaneous metastases is termed "carcinoma telangiectoides" and develops due to the presence of malignant cells in the dermal capillaries where they proliferate leading to the enlargement of these vessels (10). Histopathological examination of the cutaneous lesions can also have various aspects including glandular, Indian pattern of malignant cells situated between collagen fibers, embolization of the malignant cells with tumoral lymphatic nests or epidermotropic patterns (3). In order to confirm the malignant origin of the skin lesion, histopathological studies should be associated with immunohistochemical staining especially with cathepsin $D$, pan cytokeratins, epithelial membrane antigen or carcinoembrionic antigen; immunoreactivity of the metastatic tumor to androgen receptor is another clue which can orientate the diagnosis $(3,18,19)$.
When it comes to the clinical aspect of the problem, the presence of skin involvement in breast cancer classifies the lesion as an IVB breast cancer.

As for the long term prognosis, patients with cutaneous metastases originating from breast cancer have a better outcome when compared to those with skin lesions originating from other primaries $(12,19,20)$. In the study conducted by Lookingbill et al involving 4020 patients with metastatic disease, $10 \%$ of them had cutaneous metastatic lesions; the main primaries were breast cancer and malignant melanoma; when studying the long term outcomes, the mean overall survival ranged between 1 months and 34 months depending on the tumor type; for cases with cutaneous metastases from breast cancer a mean overall survival of 31 months was reported (19).

In a similar study conducted by Schoenlaub et al in the Laboratoire d'Histo-pathologie Cutanée of Strasbourg between 1950 to 1996, 228 patients with cutaneous metastasis were introduced. The median survival after cutaneous metastasis was 6.5 months. Median survival was calculated according to the primary malignancy and was 13.8 months for breast carcinoma, 13.5 months for melanoma and only 2.9 months for lung carcinoma. The outcome of patients with cutaneous metastasis of lung carcinoma was worse than those with melanoma $(p<10-4)$ and breast cancer $(p<10-4)(20)$.

In the study conducted by Hu et al in 2008 involving 141 patients with cutaneous metastases, 51 patients had skin lesions originating from breast cancer. For patients with breast cancer withskin metastases only group the 1-, 3-, 5-, and 10-year cumulativeoverall survival rates were $79 \%, 51 \%, 37 \%$ and $11 \%$, respectively. In the same subgroup 7 he median survival is 42.15 months while the mean survival is 57.43 months (12).

Similarly to our case, Harrison et al reported the case of a 56 year old patient diagnosed with inflammatory breast cancer with skin metastases to her back and contralateral breast. She was submitted to neo-adjuvant chemotherapy followed by bilateral modified radical mastectomy and radiation therapy; postoperatively she remained disease free based on PET imaging one year after the initial diagnosis (21).

\section{CONCLUSIONS}

Although the presence of synchronous cutaneous metastases is a sign of poor prognosis for patients with breast cancer, neoadjuvant chemotherapy followed by radical surgery and radiation therapy might be performed with good results. Contrarily to other malig- 
nancies which can develop cutaneous metastases, breast cancer with skin involvement has a better outcome, with significantly higher overall survival rates.

\section{Acknowledgement}

This paper was co-financed from the European Social Fund, through POSDRU 2007-2013, Prioritary axis no. 1, „Education and formation supporting economical development and aknowledgment based society development - major domain of intervention 1.5 "Doctoral and post-doctoral programs supporting research".

CERO - carrier profile: - Romanian researcher

Financial contract: POSDRU/159/1.5/S/135760

\section{REFERENCES}

1. Naser AMB, Zaki MS, Brunner M, Wollina V, Zouboulis CC. Cutaneous Metastasis In Internal Malignancy. Egyptian Dermatol Online J 2007; 3:1.

2. Helm TN, Lee TC. Metastatic carcinoma of the skin. emedicine, http://www.emedicine.com/derm/topic263.htm [last accessed on 2008 May 28].

3. Mordenti C, Peris K, Concetta Fargnoli M, Cerroni $L$ and Chimenti S. Cutaneous metastatic breast carcinoma. Acta Derm Venereol 2000;9:4 http://www1.mf.uni-lj.si/acta-apa/ acta-apa-00-4/mordenti.html [last accessed on 2008 May 28].

4. Prabhu S, Pai SB, Handattu S, Kudur MH, Vasanth V. Cutaneous metastases from carcinoma breast: the common and the rare. Indian J Dermato Venereol Leprol 2009 Sep;75(5):499-502.

5. Spencer PS, Helm TN. Skin metastases in cancer patients. Cutis 1987 Feb;39(2):119-21.

6. Krathen RA, Orengo IF, Rosen T. Cutaneous metastasis: a meta-analysis of data. South Med J 2003 Feb;96(2):164-7.
7. Brownstein MH, Helwig EB. Metastatic tumors of the skin. Cancer 1972 May;29(5):1298-307.

8. American Cancer Society. Breast Cancer Facts \& Figures 2005-2006. Atlanta: American Cancer Society, Inc.

9. Lund-Nielsen B, Muller K, Adamsen L. Malignant wounds in women with breast cancer: feminine and sexual perspectives. J Clin Nurs 2005 Jan;14(1):56-64.

10. Marneros AG, Blanco F, Husain S, Silvers DN, Grossman ME. Classification of cutaneous intravascular breast cancer metastases based on immunolabeling for blood and lymph vessels. J Am Acad Dermatol 2009 Apr;60(4):633-8.

11. Hussein MR. Skin metastasis: a pathologist's perspective. J Cutan Pathol 2010 Sep;37(9):e1-20.

12. Hu SC, Chen GS, Lu YW, Wu CS, Lan CC. Cutaneous metastases from different internal malignancies: a clinical and prognostic appraisal. J Eur Acad Dermatol Venereol 2008 Jun;22(6):735-40.

13. Kalmykow B, Walker $\mathrm{S}$. Cutaneous metastases in breast cancer. Clin $\mathrm{J}$ Oncol Nurs 2011 Feb;15(1):99-101.

14. Tomasini C, Soro E, Pippione M. Eyelid swelling: think of metastasis of histiocytoid breast carcinoma. Dermatology 2002;205(1):63-6.

15. Gugle A, Malpathak V, Zawar V, Deshmukh M, Kote R. Carcinoma erysipeloides: an unusual presentation mimicking radiation dermatitis. Dermatol Online J 2008;14(2):26.

16. Santos-Juanes J, Lopez-Escobar M, Palicio NV, Rivas BM, Galache C, Sancehz del Rio, et al. Zosteriform cutaneous metastasis from a breast carcinoma. Med Cutan Iber Lat Am 2007;35:89-93.

17. Lever LR, Holt PJ. Carcinoma erysipeloides. Br J Dermatol 1991 Mar;124(3):279-82.

18. Cox SE, Cruz PD, Jr. A spectrum of inflammatory metastasis to skin via lymphatics: three cases of carcinoma erysipeloides. J Am Acad Dermatol 1994 Feb; 30(2 Pt 2):304-7.

19. Lookingbill DP, Spangler N, Helm KF. Cutaneous metastases in patients with metastatic carcinoma: a retrospective study of 4020 patients. J Am Acad Dermatol 1993 Aug;29(2 Pt 1):228-36.

20. Schoenlaub P, Sarraux A, Grosshans E, Heid E, Cribier B. [Survival after cutaneous metastasis: a study of 200 cases]. Ann Dermatol Venereol 2001 Dec;128(12):1310-5.

21. Harrison AM, Zendejas B, Ali SM, Scow JS, Farley DR. Lessons learned from an unusual case of inflammatory breast cancer. J Surg Educ 2012 May;69(3):350-4. 